\title{
Synergistic interaction between MEK inhibitor and gefitinib in EGFR-TKI-resistant human lung cancer cells
}

\author{
SUXIA LI, SUXIU CHEN, YIYAN JIANG, JIEFAN LIU, XIAOLEI YANG and SHICHAO QUAN \\ Department of Tumor Rehabilitation, First Hospital, Wenzhou Medical University, Wenzhou, Zhejiang 325000, P.R. China
}

Received October 16, 2014; Accepted July 9, 2015

DOI: $10.3892 / \mathrm{ol} .2015 .3577$

\begin{abstract}
With the increasing use of epidermal growth factor receptor tyrosine kinase inhibitor (EGFR-TKIs) in patients with advanced non-small cell lung cancer (NSCLC), acquired resistance has become a major clinical problem. A combination of different signaling pathway inhibitors is a promising strategy to overcome this. In the present study, the mitogen-activated protein kinase kinase $1 / 2$ inhibitor, AZD6244, was used in combination with gefitinib to investigate the efficacy of this treatment in NSCLC cell lines, particularly in gefitinib-resistant cells. The EGFR-TKI-sensitive PC-9 (mutant EGFR/wild-type K-Ras) and EGFR-TKI-resistant A549 (wild-type EGFR/mutant K-Ras) human NSCLC cell lines were treated with AZD6244 alone, gefitinib alone or the combination of the two drugs, and the effects were evaluated using cell proliferation assays, with alterations in signaling pathways analyzed by western blotting. It was found that the growth inhibitory effect of combination treatment with gefitinib and AZD6244 was greater than that of gefitinib alone in the EGFR-TKI-resistant A549 cells. Treatment of A549 cells with gefitinib alone reduced the expression level of the activated form of Akt, and the combination of the two drugs showed stronger inhibition of phosphorylated-Akt and phosphorylated-extracellular signal-regulated kinases. The data showed that the combination of AZD6244 and gefitinib exhibited dose-dependent synergism in gefitinib-resistant NSCLC cells. Thus, a preclinical rationale exists for the use of AZD6244 to enhance the efficacy of gefitinib in patients with EGFR-TKI-resistant NSCLC.
\end{abstract}

\section{Introduction}

Non-small cell lung cancer (NSCLC) remains the cancer type with the highest mortality in the United States, with

Correspondence to: Dr Suxia Li, Department of Tumor Rehabilitation, First Hospital, Wenzhou Medical University, 2 Fuxue Lane, Wenzhou, Zhejiang 325000, P.R. China

E-mail: drlisx@163.com

Key words: non-small cell lung cancer, epidermal growth factor receptor tyrosine kinase inhibitor, gefitinib, MEK1/2 inhibitor, AZD6244
$>150,000$ fatalities anticipated in 2013 (1). The majority of NSCLC patients are diagnosed with the advanced stage of the disease, leading to a poor prognosis, with a median survival time of only 10-12 months $(2,3)$. Advances in the molecular characterization of NSCLC, have resulted in the use of novel drugs that are able to target oncogenic mutations. The efficacy of NSCLC therapy has increased following the development of epidermal growth factor receptor tyrosine kinase inhibitors (EGFR-TKIs), such as gefitinib and erlotinib $(1,4)$. EGFR-TKIs attenuate EGFR autophosphorylation and the subsequent activation of downstream pathways that regulate cell survival and growth, including the phosphoinositide 3-kinase (PI3K)-Akt-mammalian target of rapamycin signal pathway and the mitogen-activated protein kinase (MAPK) cascade [Ras-Raf-mitogen-activated protein kinase kinase (MEK)-extracellular signal-regulated kinases (ERK)] (5).

Despite EGFR-TKIs being an effective clinical therapy in patients with advanced NSCLC and EGFR-activated mutations, all patients will eventually develop acquired resistance to these drugs. Following continuous use of TKIs for 9-11 months, the majority of patients who initially respond to the treatment subsequently experience progression of the disease (6); $\sim 50 \%$ of those with an initial response to EGFR-TKIs acquire resistance to these drugs. The challenge of overcoming the resistance to TKIs in clinical practice requires a novel method.

A range of mechanisms have been proposed to underlie the acquired resistance to EGFR-TKIs. For example, a second mutation in the EGFR kinase domain, particularly a T790M mutation in exon 20, accounts for $50 \%$ of this acquired resistance (7); the T790M gate keeper mutation decreases the binding of EGFR-TKI to the mutant EGFR (8). Another $20 \%$ of patients develop TKI acquired resistance results from MET amplification (9); MET amplification-induced persistent activation of the PI3K-Akt pathway via a HER3-dependent pathway is believed to be the common downstream pathway of the acquired resistance of NSCLC.

Moreover, the presence of the K-Ras mutation is associated with the primary resistance to EGFR-TKIs. In total, $15-30 \%$ of patients with NSCLC exhibit a gain of function mutation in the K-Ras gene, resulting in a failure to respond to EGFR-TKI treatment. In NSCLC, the activation of K-Ras leads to the overexpression of ERK1/2 via the Raf/MEK/ERK signaling pathway. Therefore, inhibiting the Raf/MEK/ERK signaling pathway would result in an improvement in the antitumor effects following TKI-acquired resistance, however, the 
function of the Raf/MEK/ERK pathway in acquired resistance in NSCLC has not been extensively examined. In the present study, the antitumor effects of gefitinib alone compared with gefitinib in combination with MEK inhibitor, AZD6244, was investigated in EGFR-TKI-resistant NSCLC cells.

\section{Materials and methods}

Drugs and cell lines. Gefitinib and AZD6244 (selumetinib) were obtained from Astrazeneca (Cheshire, UK). Gefitinib and AZD6244 were prepared in dimethyl sulfoxide to obtain a stock solution of $10 \mathrm{mM}$. The EGFR-TKI-sensitive PC-9 (mutant EGFR/wild-type K-Ras) and EGFR-TKI-resistant A549 (wild-type EGFR/mutant K-Ras) human NSCLC cell lines were purchased from the American Type Culture Collection (Manassas, VA, USA) and maintained in RPMI-1640 medium (Hyclone, Logan, UT, USA), supplemented with $10 \%$ heat-inactivated fetal bovine serum (Hyclone), penicillin $(100 \mathrm{U} / \mathrm{ml})$, streptomycin $(100 \mu \mathrm{g} / \mathrm{ml})$ and L-glutamine $(2 \mathrm{mM})$ at $37^{\circ} \mathrm{C}$ in a $5 \% \mathrm{CO}_{2}$ atmosphere, and then harvested with trypsin-EDTA when the cells reached exponential growth.

Anti-proliferative effects of agents. The anti-proliferative effects of gefitinib and AZD6244 as single agents on A549 and PC-9 cells were evaluated by MTT assay. The cells were cultured in 96-well plates, with 4,000 A549 cells per well or 6,000 PC-9 cells per well. Gefitinib was added at concentrations of 10, 20, 30, 40 and $50 \mu \mathrm{mol} / 1$ after $24 \mathrm{~h}$. AZD6244 was added at concentrations of $0.001,0.01,0.1,1$ and $10 \mu \mathrm{mol} / 1$ after $24 \mathrm{~h}$. The half maximal inhibitory concentration $\left(\mathrm{IC}_{50}\right)$ was determined following $72 \mathrm{~h}$ of exposure to the drug compared with unexposed control cells. Once the cells had been exposed to each drug for $72 \mathrm{~h}$ in the 96-well plates, $20 \mathrm{ml}$ MTT solution was added to each well. The optical density (OD) of each well was measured at $490 \mathrm{~nm}$ following incubation for $4 \mathrm{~h}$. The percentage of cell growth inhibition resulting from each drug was calculated as: $\left[\left(\mathrm{OD}_{490}\right.\right.$ of control cells - $\mathrm{OD}_{490}$ of treated cells) / $\mathrm{OD}_{490}$ of control cells] x 100 . This assay was repeated for $>3$ independent experiments.

Western blotting. The cells were treated with gefitinib plus AZD6244 at various concentrations. To evaluate downstream signaling, treated cells were harvested, washed with phosphate-buffered saline and lysed. Western blotting was performed as described previously (10). The primary antibodies were as follows: $\beta$-actin (rabbit anti-human monoclonal; cat no. 8457; 1:1,000; Cell Signaling Technology, Beverly, MA, USA), EGFR (rabbit anti-human monoclonal; cat no. ab32562; 1:5,000) and its phosphorylated (p) components pY1173 (rabbit anti-human monoclonal; cat no. ab32578; 1:1,000), Akt (mouse anti-human monoclonal; cat no. ab124341; 1:1,000) and pAkt (rabbit anti-human monoclonal; cat no. 4060; 1:2,000), ERK (rabbit anti-human polyclonal; cat no. 9102; 1:1,000) and pERK (rabbit anti-human monoclonal; cat no. 4370; 1:2,000) (Cell Signaling Technology Inc., Danvers, MA, USA). Proteins were visualized with a horseradish peroxidase-coupled secondary antibody (anti-rabbit IgG; cat no. 7071; 1:5,000) obtained from Cell Signaling Technology Inc. Membranes were then washed again three times for $10 \mathrm{~min}$ each with Tris-buffered saline with Tween 20. Target protein bands were visualized using
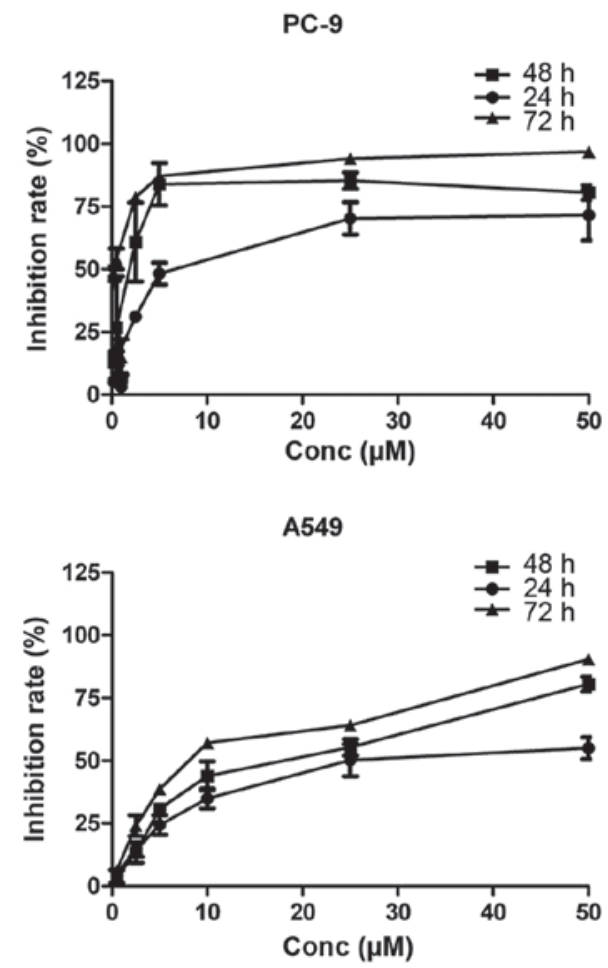

Figure 1. Anti-proliferative effects of gefitinib as the single agent on EGFR-TKI-sensitive PC-9 (mutant EGFR/wild-type K-Ras) and EGFR-TKI-resistant A549 (wild-type EGFR/mutant K-Ras) non-small cell lung cancer cell lines. EGFR-TKI, epidermal growth factor receptor tyrosine kinase inhibitor.<smiles>Cn1cnc2c(F)c(Nc3ccc(Br)cc3Cl)c(C(=O)NOCCO)cc21</smiles>

Figure 2. Structure of AZD6244 (selumetinib).

the enhanced chemiluminescence method. The intensity of the bands was quantified using the Tanon GIS system (Tanon, Shanghai, China) and the data were normalized to the $\beta$-actin loading controls. All western blotting analyses were performed three times.

Statistical analysis. Results obtained from a minimum of three independent experiments are expressed as the mean \pm standard deviation. Student's t-test and a one-way analysis of variance test were used to determine the differences between control and treatment groups. Data analyses were performed using SPSS statistical software (version 15.0; SPSS, Inc., Chicago, IL, USA). $\mathrm{P}<0.05$ was used to indicate a statistically significant difference.

\section{Results}

Sensitivity of NSCLC cell lines to EGFR and MEK1/2 inhibitors. MTT assays were used to evaluate the anti-proliferative effects of gefitinib $(0,0.25,0.5,2.5,5,25$ and $50 \mu \mathrm{M})$ as a single agent 
PC-9

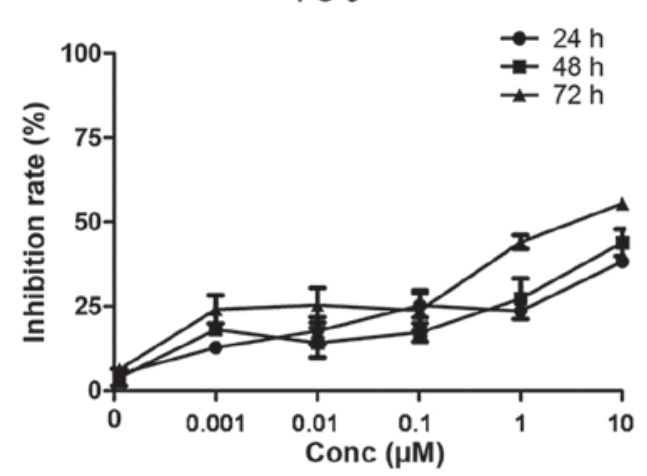

A549

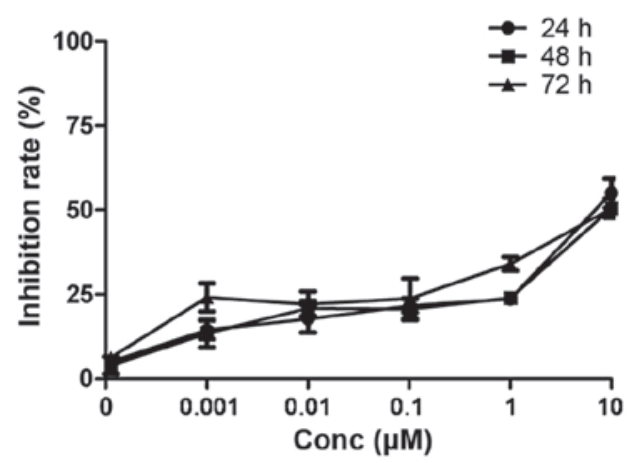

Figure 3. Anti-proliferative effects of AZD6244 as the single agent on EGFR-TKI-sensitive PC-9 (mutant EGFR/wild-type K-Ras) and EGFR-TKI-resistant A549 (wild-type EGFR/mutant K-Ras) non-small cell lung cancer cell lines. EGFR-TKI, epidermal growth factor receptor tyrosine kinase inhibitor.

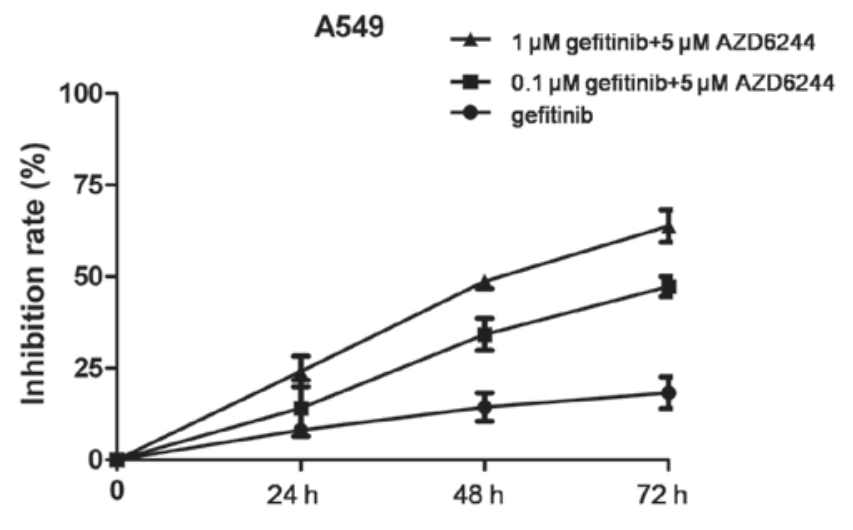

Figure 4. Co-treatment with gefitinib and mitogen-activated protein kinase kinase 1/2 inhibitor, AZD6244, overcomes gefitinib resistance in epidermal growth factor receptor (EGFR) tyrosine kinase inhibitor-resistant A549 (wild-type EGFR/mutant K-Ras) cells.

on EGFR-TKI-sensitive PC-9 (mutant EGFR/wild-type K-Ras) and EGFR-TKI-resistant A549 (wild-type EGFR/mutant K-Ras) NSCLC cell lines. Dose-dependent growth inhibitory effects of gefitinib were observed in the two NSCLC cell lines (Fig. 1). It was shown that the EGFR-TKI-sensitive PC-9 cells were more sensitive to gefitinib than the EGFR-TKI-resistant A549 cells. The $\mathrm{IC}_{50}$ in the PC-9 and A549 cells after $72 \mathrm{~h}$ was 4.1 and $10.4 \mu \mathrm{M}$, respectively.

The MEK1/2-dependent proliferation of the NSCLC cell lines was examined by treating cells with the newly developed MEK1/2 inhibitor, AZD6244 (AstraZeneca) (Fig. 2). EGFR-TKI-sensitive PC-9 (mutant EGFR/wild-type K-Ras) and EGFR-TKI-resistant A549 (wild-type EGFR/mutant K-Ras) NSCLC cell lines were selected to test the inhibitory effect of AZD6244. Cell proliferation was analyzed by MTT assay in cells treated with $0.001,0.01,0.1,1$ and $10 \mu \mathrm{M}$ of the AZD6244. The results showed that the lung cancer cell lines tested were all resistant to AZD6244 monotherapy (Fig. 3), with an $\mathrm{IC}_{50}$ of $>10 \mu \mathrm{M}$. Thus, the EGFR-TKI-resistant A549 (wild-type EGFR/mutant K-Ras) NSCLC cell line was selected for the combination treatment experiments.

Synergistic growth inhibitory effect of gefitinib and AZD6244 in NSCLC cells. Since the KRAS-RAF-MEK-ERK cascade

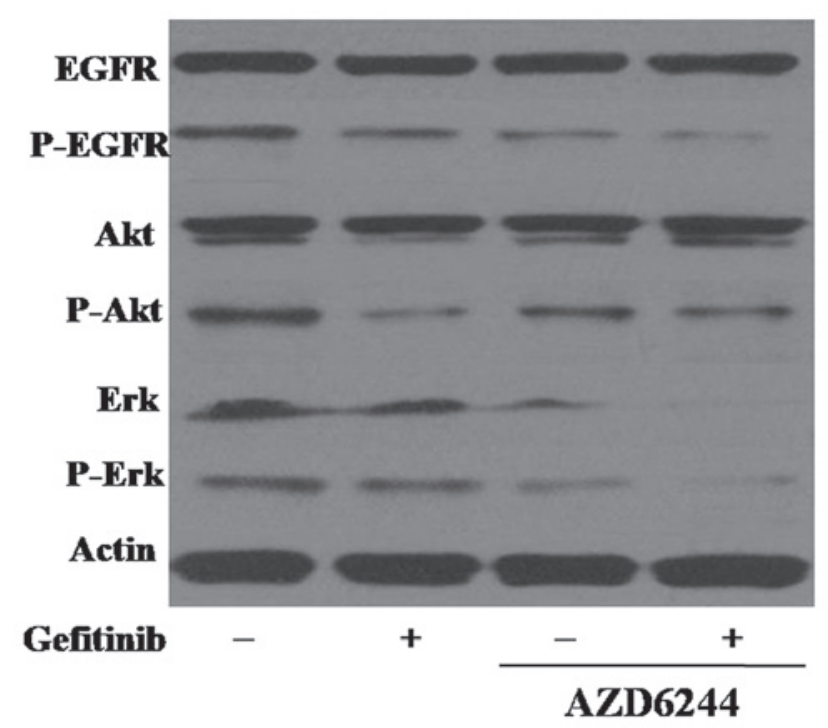

Figure 5. Combination treatment with gefitinib and mitogen-activated protein kinase kinase (MEK)1/2 inhibitor, AZD6244, significantly inhibits MEK/extracellular signal-regulated kinases (ERK) and phosphoinositide 3-kinase (PI3K)/Akt pathways. P, phosphorylated; EGFR, epidermal growth factor receptor.

is the main downstream path way of EGFR signaling, we hypothesized that the dual inhibition of EGFR and MEK1/2 may enhance the therapeutic efficacy of each agent. Therefore, the effect of gefitinib $(0.1$ or $1 \mu \mathrm{M})$, alone or in combination with $5 \mu \mathrm{M}$ AZD6244, was examined. The growth inhibitory effect of the combination treatment was greater than that of gefitinib alone in the EGFR-TKI-resistant A549 cells (Fig. 4).

Effects of AZD6244 in combination with gefitinib on the EGFR signaling pathway. The effects of AZD6244 and gefitinib on the EGFR pathway in gefitinib-sensitive and -resistant cell lines were determined using western blot analysis. In gefitinib-sensitive PC-9 cells, $0.25 \mu \mathrm{M}$ gefitinib was sufficient to completely dephosphorylate EGFR, but the inhibitory effect of gefitinib on the phosphorylation of EGFR in the EGFR-TKI-resistant A549 cells was lower than that in the PC-9 cells. The combination treatment of gefitinib and AZD6244 in the A549 cells increased the inhibition of EGFR 
phosphorylation, suggesting that gefitinib in combination with AZD6244 demonstrated superior efficacy in gefitinib-resistant NSCLC cells.

To further assess the potential synergistic mechanisms of gefitinib and AZD6244, the effects of a combination of the two agents on the downstream Akt and ERK signaling pathways were detected by western blot analysis in the A549 cells. As the MEK/ERK and PI3K/Akt pathways are essential for cell proliferation and survival, the effect of combined treatment with gefitinib and AZD6244 on Akt phosphorylation was investigated. It was found that the treatment of A549 cells with gefitinib alone reduced the expression level of the activated form of Akt, and that the combination of the two drugs showed stronger inhibition of Erk and p-Erk (Fig. 5).

\section{Discussion}

Despite significant advances in the development of therapeutic agents against lung cancer, the mortality rate of lung cancer patients remains high. Recently, targeted anticancer drugs, including EGFR-TKIs, have been approved for the treatment of NSCLC (10). However, not all patients benefit from this therapy due to primary or acquired resistance, which are usually caused by the activation of alternative signaling pathways.

The mechanisms of resistance to EGFR inhibitors may be varied, with one of the most important mechanisms being the activation of signaling pathways downstream of EGFR. Overexpression of downstream effectors, including PI3K/Akt, results in the persistent activation of the PI3K/Akt and MAPK pathways, and the consequent development and maintenance of an EGFR-resistant phenotype (11). Therefore, inhibiting EGFR alone may not be sufficient for antitumor effects, and the development of a combination of different signaling pathway inhibitors is a promising strategy in NSCLC patients.

When considering recent promising studies on highly selective small molecules, MEK1/2 inhibitors have been developed and have shown clinical activity in a number of malignancies, including NSCLC $(12,13)$. In the present study, the MEK1/2 inhibitor, AZD6244, in combination with gefitinib was selected to investigate the efficacy of this treatment in NSCLC cell lines, particularly in gefitinib-resistant cells. The present study was performed in EGFR-TKI-sensitive PC-9 (EGFR mutant/wild-type K-Ras) and EGFR-TKI-resistant A549 (wild-type EGFR/mutant K-Ras) human NSCLC cells. Synergy was observed for the combination of gefitinib and varying doses of AZD6244 in gefitinib-resistant cell lines. The results reported in the present study demonstrate that the combined inhibition of EGFR and MEK results in a more effective grow th blockade in NSCLC cell lines compared with either agent alone. The strongest synergism in the NSCLC cells was observed upon simultaneous administration of gefitinib and AZD6244 in the two cell lines.

ERK, the only known target of MEK1/2, has multiple targets, which are involved in cell proliferation, survival, mitosis and migration (14,15). AZD6244 is a potent, selective, and orally available MEK1/2 inhibitor. Previous studies have shown that the inhibition of MEK1/2 can induce apoptosis by inhibiting ERK-mediated B-cell lymphoma 2 phosphorylation and stabilization $(16,17)$. Currently available MEK1/2 inhibitors have shown moderate single-agent activity in various tumors (18). The inability of MEK inhibitors to suppress the phosphorylation of MEK1/2 and ERK1/2 completely indicates that ERK activity may be regulated by multiple signaling pathways and that MEK1/2 inhibitors could be combined with standard chemotherapy drugs. A recent study showed that AZD6244 and docetaxel have a synergistic effect in advanced KRAS-mutated NSCLC. In the present study, it was found that the treatment of A549 cells with gefitinib alone reduced the expression level of the activated form of Akt, and that the combination of the two drugs showed stronger inhibition of Erk and p-Erk.

The present study provided evidence that a combination treatment with gefitinib and AZD6244 is effective for overcoming acquired EGFR-TKI resistance in lung cancer cells through the inhibition of ERK activity. The study suggested that EGFR-TKI combined with MEK1/2 inhibitors, such as AZD6244, may be beneficial to those patients with NSCLC who develop a primary or acquired resistance to EGFR-TKIs.

\section{Acknowledgements}

This study was supported by a grant from the Wenzhou Science and Technology Bureau (no. Y20130041).

\section{References}

1. Mok TS, Wu YL, Thongprasert S, Yang CH, Chu DT, Saijo N, Sunpaweravong P, Han B, Margono B, Ichinose Y, et al: Gefitinib or carboplatin-paclitaxel in pulmonary adenocarcinoma. N Engl J Med 361: 947-957, 2009.

2. Scagliotti GV, Parikh P, von Pawel J, Biesma B, Vansteenkiste J, Manegold C, Serwatowski P, Gatzemeier U, Digumarti R, Zukin M, et al: Phase III study comparing cisplatin plus gemcitabine with cisplatin plus pemetrexed in chemotherapy-naive patients with advanced-stage non-small-cell lung cancer. J Clin Oncol 26: 3543-3551, 2008.

3. Sandler A, Gray R, Perry MC, Brahmer J, Schiller JH, Dowlati A, Lilenbaum R and Johnson DH: Paclitaxel-carboplatin alone or with bevacizumab for non-small-cell lung cancer. N Engl J Med 355: 2542-2550, 2006.

4. Zhou C, Wu YL, Chen G, Feng J, Liu XQ, Wang C, Zhang S, Wang J, Zhou S, Ren S, et al: Erlotinib versus chemotherapy as first-line treatment for patients with advanced EGFR mutation-positive non-small-cell lung cancer (OPTIMAL, CTONG-0802): A multicentre, open-label, randomised, phase 3 study. Lancet Oncol 12: 735-742, 2011.

5. Ono M and Kuwano M: Molecular mechanisms of epidermal growth factor receptor (EGFR) activation and response to gefitinib and other EGFR-targeting drugs. Clin Cancer Res 12: 7242-7251, 2006.

6. Costa DB, Kobayashi S, Tenen DG and Huberman MS: Pooled analysis of the prospective trials of gefitinib monotherapy for EGFR-mutant non-small cell lung cancers. Lung Cancer 58: 95-103, 2007.

7. Pao W, Miller VA, Politi KA, Riely GJ, Somwar R, Zakowski MF, Kris MG and Varmus H: Acquired resistance of lung adenocarcinomas to gefitinib or erlotinib is associated with a second mutation in the EGFR kinase domain. PLoS Med 2: e73, 2005.

8. Sos ML, Rode HB, Heynck S, Peifer M, Fischer F, Klüter S, Pawar VG, Reuter C, Heuckmann JM, Weiss J, et al: Chemogenomic profiling provides insights into the limited activity of irreversible EGFR Inhibitors in tumor cells expressing the T790M EGFR resistance mutation. Cancer Res 70: 868-874, 2010.

9. Engelman JA, Zejnullahu K, Mitsudomi T, Song Y, Hyland C, Park JO, Lindeman N, Gale CM, Zhao X, Christensen J, et al: MET amplification leads to gefitinib resistance in lung cancer by activating ERBB3 signaling. Science 316: 1039-1043, 2007.

10. Zhang H, Xu Y, Filipovic A, Lit LC, Koo CY, Stebbing J and Giamas G: SILAC-based phosphoproteomics reveals an inhibitory role of KSR1 in p53 transcriptional activity via modulation of DBC1. Br J Cancer 109: 2675-2684, 2013. 
11. Li J,Pan YY and Zhang Y: Synergisticinteraction between sorafenib and gemcitabine in EGFR-TKI-sensitive and EGFR-TKI-resistant human lung cancer cell lines. Oncol Lett 5: 440-446, 2013.

12. Kim KB, Kefford R, Pavlick AC, Infante JR, Ribas A, Sosman JA, Fecher LA, Millward M, McArthur GA, Hwu P, et al: Phase II study of the MEK1/MEK2 inhibitor Trametinib in patients with metastatic BRAF-mutant cutaneous melanoma previously treated with or without a BRAF inhibitor. J Clin Oncol 31: 482-489, 2013

13. Metro G, Chiari R, Baldi A, De Angelis V, Minotti V and Crinò L: Selumetinib: A promising pharmacologic approach for KRAS-mutant advanced non-small-cell lung cancer. Future Oncol 9: 167-177, 2013.

14. Meloche S and Pouysségur J: The ERK1/2 mitogen-activated protein kinase pathway as a master regulator of the G1- to S-phase transition. Oncogene 26: 3227-3239, 2007.

15. Balmanno K and Cook SJ: Tumour cell survival signalling by the ERK1/2 pathway. Cell Death Differ 16: 368-377, 2009.
16. Bodoky G, Timcheva C, Spigel DR, La Stella PJ, Ciuleanu TE, Pover G and Tebbutt NC: A phase II open-label randomized study to assess the efficacy and safety of selumetinib (AZD6244 [ARRY-142886]) versus capecitabine in patients with advanced or metastatic pancreatic cancer who have failed first-line gemcitabine therapy. Invest New Drugs 30: 1216-1223, 2012.

17. Hainsworth JD, Cebotaru CL, Kanarev V, Ciuleanu TE, Damyanov D, Stella P, Ganchev H, Pover G, Morris C and Tzekova V: A phase II, open-label, randomized study to assess the efficacy and safety of AZD6244 (ARRY-142886) versus pemetrexed in patients with non-small cell lung cancer who have failed one or two prior chemotherapeutic regimens. J Thorac Oncol 5: 1630-1636, 2010

18. Kim EY, Kim A, Kim SK and Chang YS: AZD6244 inhibits cisplatin-induced ERK1/2 activation and potentiates cisplatin-associated cytotoxicity in K-ras G12D preclinical models. Cancer Lett 358: 85-91, 2015. 